\title{
Differentiation of human adipose-derived stem cells into "brite" (brown-in-white) adipocytes
}

\author{
Didier F. Pisani ${ }^{1,2}$, Mansour Djedaini ${ }^{1,2}$, Guillaume E. Beranger ${ }^{1,2}$, Christian Elabd $^{1,2,3}$, Marcel Scheideler ${ }^{4}$, \\ Gérard Ailhaud ${ }^{1,2}$ and Ez-Zoubir Amri ${ }^{1,2}{ }^{\text {* }}$
}

1 Institut de Biologie du Développement et Cancer, Université de Nice Sophia-Antipolis, Nice, France

${ }^{2}$ CNRS UMR 6543, Institut de Biologie du Développement et Cancer, Nice, France

${ }^{3}$ Department of Bioengineering, Institute for Quantitative Biosciences, University of California Berkeley, Berkeley, CA, USA

${ }^{4}$ Institute for Genomics and Bioinformatics, Graz University of Technology, Graz, Austria

\section{Edited by:}

Patrick Seale, University of

Pennsylvania, USA

Reviewed by:

Miguel Lopez, University of Santiago de Compostela, Spain

Darlene Evans Berryman, Ohio

University, USA

Anthony Scime, York University,

Canada

Rana Gupta, Dana-Farber Cancer

Institute, USA

${ }^{*}$ Correspondence:

Ez-Zoubir Amri, CNRS UMR 6543,

Institut de Biologie du

Développement et Cancer, Université de Nice Sophia-Antipolis, 28 Avenue de Valombrose 06107, Nice Cedex 2 ,

France.

e-mail:amri@unice.fr
It is well established now that adult humans possess active brown adipose tissue (BAT) which represents a potential pharmacological target to combat obesity and associated diseases. Moreover thermogenic brown-like adipocytes ("brite adipocytes") appear also in mouse white adipose tissue (WAT) upon $\beta 3$-adrenergic stimulation. We had previously shown that human multipotent adipose-derived stem cells (hMADS) are able to differentiate into cells which exhibit the key properties of human white adipocytes, and then to convert into functional brown adipocytes upon PPAR $\gamma$ activation. In light of a wealth of data indicating that thermogenic adipocytes from BAT and WAT have a distinct cellular origin, we have characterized at the molecular level UCP1 positive hMADS adipocytes from both sexes as brite adipocytes. Conversion of white to brown hMADS adipocytes is dependent on PPAR $\gamma$ activation with rosiglitazone as the most potent agonist and is inhibited by a PPAR $\gamma$ antagonist. In contrast to mouse cellular models, hMADS cells conversion into brown adipocytes is weakly induced by BMP7 treatment and not modulated by activation of the Hedgehog pathway. So far no primary or clonal precursor cells of human brown adipocytes have been obtained that can be used as a tool to develop therapeutic drugs and to gain further insights into the molecular mechanisms of brown adipogenesis in humans. Thus hMADS cells represent a suitable human cell model to delineate the formation and/or the uncoupling capacity of brown/brite adipocytes that could help to dissipate caloric excess intake among individuals.

Keywords: stem cells, rosiglitazone, adipocyte, differentiation, UCP1, brite adipocyte, BAT, WAT

\section{INTRODUCTION}

Obesity has reached epidemic proportions globally, with more than one billion adults overweight and at least 300 million of them clinically obese. In addition, at least 155 million children worldwide are overweight or obese, according to the International Obesity Task Force (Hossain et al., 2007). Obesity constitutes a substantial risk factor for hypertension, type 2 diabetes, and cardiovascular diseases implying tremendous burdens for the public health care system. White adipose tissue (WAT) plays a central role in the control of energy homeostasis (Ailhaud et al., 1992; Rosen and Spiegelman, 2006). In contrast to WAT, brown adipose tissue (BAT) is specialized in adaptive thermogenesis in which the uncoupling protein 1 (UCP1) plays a key role (Golozoubova et al., 2006). The involvement of BAT in diet-induced thermogenesis has been recently shown in UCP1-ablated mice raised at thermoneutrality (Feldmann et al., 2009). Of utmost interest, recent data show that active BAT is indeed present as discrete and small depots in healthy adult individuals (Nedergaard et al., 2007; Cypess et al., 2009; Saito et al., 2009; Van Marken Lichtenbelt et al., 2009; Virtanen et al., 2009; Zingaretti et al., 2009) and represents a new target to fight obesity (Fruhbeck et al., 2009; Whittle et al., 2011).
At the cellular level, a myogenic signature of brown adipocytes and cell sorting of muscle and WAT derived progenitors favor a distinct origin from that of white adipocytes (Timmons et al., 2007; Crisan et al., 2008). In vivo lineage studies showed that brown adipocytes from brown-fat depots share a common developmental origin with myoblasts (Seale et al., 2008). However, islands of brown adipocytes are also detected in white fat depots of rodents following chronic exposure to cold or pharmacological stimulation of $\beta 3$-adrenoreceptor ( $\beta 3-\mathrm{AR})$. There is indeed evidence that white adipocytes can be converted to brown-like fat cells (Tiraby et al., 2003; Cinti, 2009a,b; Barbatelli et al., 2010). Within traditional WAT depots, a particular type of adipocytes occurs in rodents, corresponding to the "brite" (brown-in-white) adipocytes. These cells express UCP1 and exhibit in vitro a thermogenic response to $\beta$-AR agonists (Petrovic et al., 2010) that could explain UCP1 expressing cells present as islets surrounded by white adipocytes (Cousin et al., 1992; Xue et al., 2009). Most importantly, in vivo, the capacity of obese mice to reduce their fat mass in response to adrenergic stimulation by cold or $\beta 3$-AR agonist does not depend upon BAT whose amount is genetically invariant among mouse strains but depends upon a genetic variability which affects the development of "brite" adipocytes within 
WAT depots (Guerra et al., 1998; Xue et al., 2007). Other external cues are favoring the brown-fat differentiation, e.g., Hedgehog signaling has been characterized as a determinant of brown-fat cell fate (Pospisilik et al., 2010) whereas among bone morphogenetic proteins (BMPs), BMP7 specifically promotes brown adipogenesis of murine multipotent mesenchymal stem cells (Tseng et al., 2008; Schulz et al., 2011).

We have reported the characterization of mesenchymal stem cells from human adipose tissue of young male and female donors [termed human multipotent adipose-derived stem cells (hMADS)] which exhibit at a clonal level a normal karyotype, selfrenewal ability, the absence of tumorigenicity (Rodriguez et al., 2004, 2005a; Zaragosi et al., 2006; Elabd et al., 2007; Fontaine et al., 2008), and are able to convert into functional brown-like adipocytes (Elabd et al., 2009). Owing to recent data showing that thermogenic adipocytes from BAT and WAT originate from distinct origin, the question raised whether UCP1-positive hMADS adipocytes from both sexes are classical brown or "brite" adipocytes. Herein we provide evidence at the molecular level that these cells share similarities with mouse brite adipocytes. However, in contrast to mouse cellular models, brown adipogenesis is only slightly induced by BMP7 treatment and not modulated by activation of the Hedgehog pathway. Thus hMADS cells represent a suitable human cell model to gain insights in the formation of brite adipocytes that could help to dissipate caloric excess intake.

\section{MATERIALS AND METHODS REAGENTS}

Cell culture media, serum, buffers, and trypsin were purchased from Lonza Verviers (Verviers, Belgium) and cell culture reagents from Sigma-Aldrich Chimie (Saint-Quentin Fallavier, France). Rosiglitazone was purchased from BertinPharma (Montigny le bretonneux, France).

\section{CELL CULTURE}

The establishment and characterization of the multipotency and self-renewal capacity of hMADS cells have been described (Rodriguez et al., 2004, 2005b; Elabd et al., 2007, 2009). In the experiments reported herein hMADS-1, 2, and 3 cells, established respectively, from the umbilical fat pad of a 31-month-old female donor, from the pubic region fat pad of a 5-year-old male donor and the prepubic fat pad of a 4-month-old male, were used between passages 16 and 35 corresponding from 35 to $100 \mathrm{popu}-$ lation doublings. Cells were seeded at a density of $4500 \mathrm{cells} / \mathrm{cm}^{2}$ in Dulbecco's modified Eagle's medium (DMEM) supplemented with $10 \%$ FCS, $2.5 \mathrm{ng} / \mathrm{ml}$ hFGF2, $60 \mu \mathrm{g} / \mathrm{ml}$ penicillin, and $50 \mu \mathrm{g} / \mathrm{ml}$ streptomycin. The medium was changed every other day and hFGF2 was removed when cells reached confluence and were triggered for differentiation at day 2 post-confluence (designated as day 0). Cells were then maintained in DMEM/Ham's F12 media supplemented with $10 \mu \mathrm{g} / \mathrm{ml}$ transferrin, $0.85 \mu \mathrm{M}$ insulin, $0.2 \mathrm{nM}$ triiodothyronine, $1 \mu \mathrm{M}$ dexamethasone, $500 \mu \mathrm{M}$ isobutylmethylxanthine. Three days later, the medium was changed (dexamethasone and isobutyl-methylxanthine were omitted) and $100 \mathrm{nM}$ rosiglitazone were added for the indicated periods. Media were then changed every other day and cells used at the indicated days. Glycerol-3-phosphate dehydrogenase (GPDH) activity measurements and Oil Red O staining were performed as described previously (Negrel et al., 1978; Bezy et al., 2005).

\section{ISOLATION AND ANALYSIS OF RNA}

Total RNA was extracted using TRI-Reagent kit (Euromedex, Souffelweyersheim, France) according to the manufacturer's instructions. Quality control for purity and integrity of RNA were tested by OD $(260 / 280 \mathrm{nM})$ measurements and ethidium bromidestained agarose analysis. Reverse transcription-polymerase chain reaction (RT-PCR) analysis was conducted as described previously (Rodriguez et al., 2004; Bezy et al., 2005; Zaragosi et al., 2006; Elabd et al., 2007). Primer sequences, designed using Primer Express software (Applied Biosystems, Courtaboeuf, France), are listed in Table 1 and were tested for their specificity, efficiency, reproducibility, and dynamic range. For quantitative PCR, final reaction volume was $20 \mu \mathrm{l}$ using SYBR green master mix (Eurogentec, Angers, France) and assays were run on an ABI Prism 7700 real-time PCR machine (PerkinElmer Life and Analytical Sciences, Boston, MA, USA). An aliquot of PCR products was analyzed on $2 \%$ ethidium bromide-stained agarose. The expression of selected genes was normalized to that of TATA-box binding protein (TBP) gene and quantified using the comparative- $\Delta \mathrm{Ct}$ method. TBP expression did not vary along the adipocyte differentiation of hMADS cells; we used also 36B4 and POLR2A genes as housekeeping genes which gave similar data. Human skeletal muscle RNA extracts were obtained from a previous study (Pisani et al., 2010).

\section{MITOCHONDRIA ANALYSIS}

Living cells were submitted to $100 \mathrm{nM}$ MitoTracker Red FM (Invitrogen) for $45 \mathrm{~min}$ at $37^{\circ} \mathrm{C}$. Cells were finally washed with prewarmed culture medium and visualized with an Axiovert microscope (Carl Zeiss, Le Pecq, France) and pictures were captured and treated with AxioVision software (Carl Zeiss).

\section{CYTOCHROME C OXIDASE ACTIVITY DETERMINATIONS}

Cells were disrupted using a polytron in $10 \mathrm{mM}$ Tris $\mathrm{pH} 8,1 \mathrm{mM}$ EDTA, and $0.25 \mathrm{M}$ Sucrose containing protease inhibitors. Cell lysate was centrifuged at $750 \mathrm{~g}$ for $10 \mathrm{~min}$ with a pellet corresponding to a nuclear-enriched fraction. The supernatant was then centrifuged at $10000 \mathrm{~g}$ for $20 \mathrm{~min}$, the pellet corresponding to a mitochondrial-enriched fraction. The mitochondrial-enriched fraction was used to determine Cytochrome c Oxidase activity according to manufacturer's instructions (Cytochrome c Oxidase Assay kit, Sigma).

\section{STATISTICAL ANALYSIS}

Data are expressed as mean values \pm SEM and are analyzed using the 2-tailed Student's $t$-test. Differences were considered statistically significant at $P \leq 0.05$.

\section{RESULTS}

\section{UCP1, 2, AND 3 EXPRESSION DURING HMADS CELL DIFFERENTIATION}

We showed previously that hMADS cells were able to differentiate into white and brown adipocytes depending on the length of activation of PPAR $\gamma$ by rosiglitazone (Elabd et al., 2009). For hMADS- 1 cells, originally established from the umbilical fat pad 
Table 1 | Sequence of primers used for gene expression analysis.

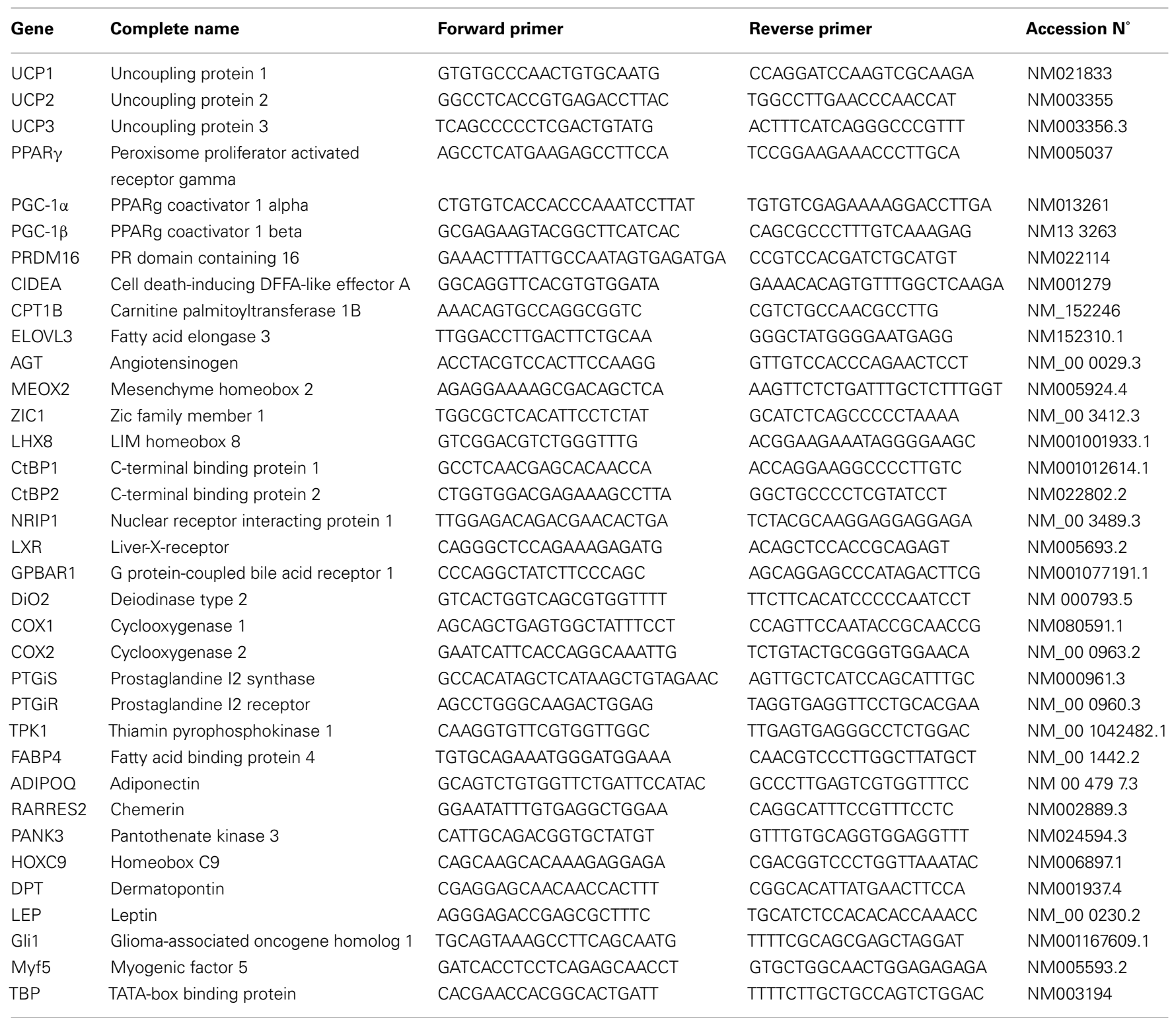

of a 31-month-old female donor, a 6-day exposure to rosiglitazone (between days 3 and 9) proved to be optimal for the cells to become white adipocytes. As shown in Figures 1A,B, further exposure of the cells to rosiglitazone did not alter the overall adipogenesis level per se since treatment between days 3 and 16 brought no change in triglyceride accumulation (Oil Red O staining) and GPDH activity. Under this condition, as shown in Figure 1C, a dramatic increase in the expression of UCP1 mRNA was observed. Interestingly, when hMADS-1 cells were first differentiated as above into white adipocytes and rosiglitazone being removed for the next 5 days, a further 2-day rosiglitazone exposure (between days 14 and 16) was sufficient to stimulate the expression of UCP1 (Figure 1C). In order to extend these observations, hMADS-2 and hMADS-3 cells, established respectively from the pubic fat pad of a 5-year-old male donor and the prepubic fat pad of a 4-month-old male, were used and gave similar results (Figure 1C). Under these conditions,
UCP1 protein could be detected by immunoblotting analysis (data not shown).

We then measured for the expression of two other members of the UCP family, UCP2 and UCP3. The expression of UCP2 mRNA was induced during adipogenesis of hMADS-1, 2, and 3 cells, and its expression was partially but not significantly enhanced by the duration of rosiglitazone treatment (Figure 1D). Interestingly, UCP3 mRNA was barely detected (100-200 times lower as compared to skeletal muscle cells), was not induced during adipogenesis and remained insensitive to rosiglitazone treatment (Figure 1E).

\section{UCP1 INDUCTION IS DEPENDENT UPON PPAR $\gamma$ ACTIVATION}

Among members of the thiazolidinedione family, rosiglitazone is known as a high-affinity ligand of PPAR $\gamma$. We therefore carried out a comparative study with other thiazolidinediones, i.e., 


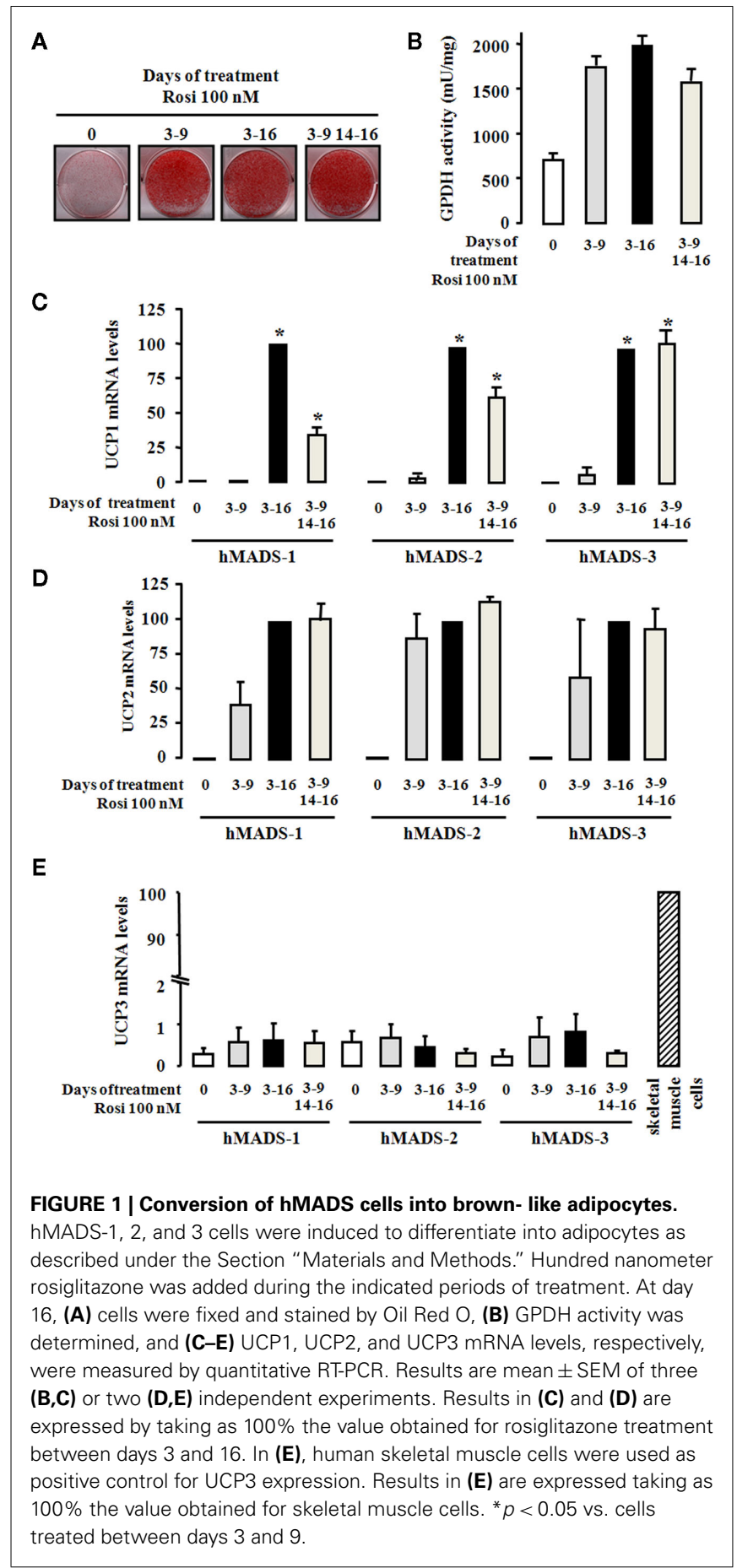

troglitazone and ciglitazone, with respect to the induction of UCP1 gene expression. For this purpose, hMADS-2 cells were first differentiated into white adipocytes as above, i.e., rosiglitazone treatment between days 3 and 9 followed by its removal for the next 5 days, were then exposed to increasing concentrations of various thiazolidinediones between days 14 and 16 . The levels of UCP1 mRNA were analyzed by quantitative RT-PCR at day 16. As shown in Figure 2A, troglitazone and ciglitazone were able to induce UCP1 expression in a dose-dependent manner.
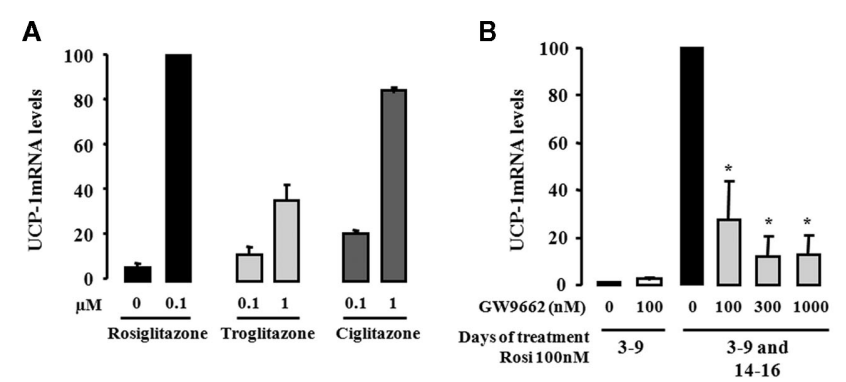

FIGURE 2 | Activation of PPAR $\boldsymbol{\gamma}$ is involved in the conversion of white to brown adipocytes. hMADS-2 cells were induced to differentiate into white adipocytes in the presence of $100 \mathrm{nM}$ rosiglitazone between days 3 and 9 , and then treated with various component as indicated between days 14 and 16. (A) Effect of various PPAR $\gamma$ agonists (Rosiglitazone, Troglitazone, and Ciglitazone) analyzed by quantitative RT-PCR of UCP1 gene expression. (B) Effect of the PPAR $\gamma$ antagonist GW9662 on UCP1 expression stimulated by rosiglitazone. Results are the mean \pm SEM of two independent experiments. Results are expressed by taking as $100 \%$ the value obtained at $100 \mathrm{nM}$ rosiglitazone. ${ }^{*} p<0.05$ vs. cells exposed to $100 \mathrm{nM}$ rosiglitazone between days 3-9 and days 14-16.

Clearly, the potency of rosiglitazone was already maximal at a dose where the effects of troglitazone and ciglitazone were weak. In order to gain further insights into the critical role of PPAR $\gamma$, we tested whether a specific PPAR $\gamma$ antagonist (GW9662) was able to abolish the effect of rosiglitazone on UCP1 expression. As above, hMADS-2 cells were first differentiated into white adipocytes, then treated or not with $100 \mathrm{nM}$ rosiglitazone in the presence or absence of the PPAR $\gamma$ antagonist. As shown in Figure 2B the rosiglitazone-induced UCP1 mRNA expression was abolished in a dose-dependent manner by GW9662. Of note, GW9662 showed no effect on untreated hMADS cells. Similar data were observed using hMADS-3 cells (data not shown). Altogether, these observations demonstrated that induction of UCP1 was dependent on $\operatorname{PPAR} \gamma$ activation and that rosiglitazone was the most efficient agonist in this process.

\section{CHARACTERIZATION OF MITOCHONDRIOGENESIS IN DIFFERENTIATED hMADS ADIPOCYTES}

As abundance of mitochondria is higher in brown as compared to white fat cells, we tested whether mitochondriogenesis was affected in the "browning" process of hMADS cells. Clearly, more mitochondria were observed in brown-like adipocytes as compared to white adipocytes (Figure 3A). This observation was further supported by quantitative RT-PCR analysis of CPT1B, a fatty acid transporter of the outer mitochondrial membrane (Figure 3B). Under these conditions, CPT1B mRNA levels were 10 -fold higher in brown-like adipocytes as compared to white adipocytes. Furthermore, the increase of CPT1B mRNA levels upon rosiglitazone treatment was similar to that observed for UCP1 (Figure 1C).

Finally, we measured the Cytochrome c oxidase activity in this fraction as an index of respiratory chain activity. As expected, Cytochrome $\mathrm{c}$ oxidase activity was higher in UCP1-containing fractions from hMADS cells chronically treated as compared to cells treated between days 3 and 9 (Figure 3C). Thus, hMADS 
A

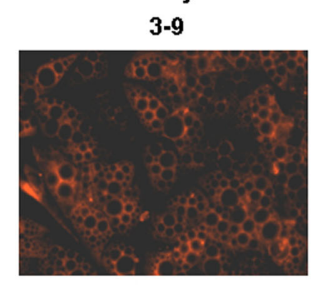

\author{
B
}

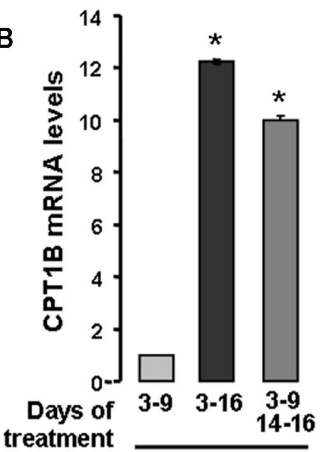

Rosi $100 \mathrm{nM}$
hMADS-1

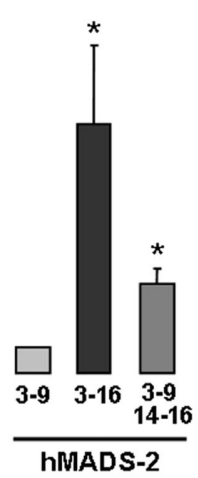

hMADS-2
FIGURE 3 | Mitochondriogenesis of hMADS cells after conversion to brown-like adipocytes. hMADS-2 cells were induced to differentiate into adipocytes in the presence of $100 \mathrm{nM}$ rosiglitazone between days 3 and 9 or between days 3 and 16. Alternatively, after removal at day 9 , rosiglitazone was re-added or not at day 14 for 2 days. (A) Mitochondria visualized by epifluorescence in living cells in the presence of the mitotracker dye, (B) CPT1B mRNA levels determined at day 16 by quantitative RT-PCR in hMADS-1, 2, and 3 cells and (C) Cytochrome $\mathrm{C}$ oxidase activity determined at day 16 . Results are mean \pm SEM $(\mathbf{B}, \mathbf{C})$ and are representative experiments (A) of three independent experiments performed on different series of cells. ${ }^{*} p<0.05$ vs. cells treated between days 3 and 9 . adipocytes expressing UCP1 display a significant increase in mitochondriogenesis accompanied by an increase in their respiratory activity; both indicating the acquisition of a brown-like phenotype. As brown-like adipocytes arise from white adipocytes, brown-like adipocytes can be defined at first sight under our conditions as brite adipocytes.

\section{ANALYSIS OF GENE EXPRESSION IN hMADS-DERIVED BRITE ADIPOCYTES}

Recently, studies of mouse model have described genes with differential expression in white vs. brown adipocytes (Vernochet et al., 2009; Petrovic et al., 2010). Therefore we aimed at analyzing the expression of a large set of these genes in white vs. brown-like hMADS adipocytes.

First, we analyzed the expression of Myf5 known to be a molecular signature common to brown-fat and skeletal muscle cells (Seale et al., 2008). Myf5 expression was not detectable in proliferating or differentiated hMADS cells originating from three donors (data not shown).

Table 2 summarized the expression values ( $\Delta \mathrm{Ct}$ referred to TBP) of different genes in hMADS cells at day 9 of adipocyte differentiation as well as day 16 of white and brite differentiation of hMADS- 2 and 3 cells. White and brite hMADS adipocytes expressed classical adipogenic markers (FABP4, ADIPOQ, RARRES2, PANK3, PPAR $\gamma$, and low level of LXR) at similar levels. In addition, hMADS brite adipocytes expressed UCP1, CPT1B, CIDEA, and ELOVL3, four genes found to be highly expressed in mouse brite adipocytes (Petrovic et al., 2010). Conversely, these cells did not express ZIC1 and LHX8, two genuine brown adipocyte specific genes (Vernochet et al., 2009). Interestingly, hMADS cells expressed PRDM16, MEOX2, and AGT at the same level in both white and brite adipocytes. With respect to white adipocyte specific genes, it is noticeable that leptin (LEP) and DPT but not HOXC9 levels decreased with brite adipocyte formation.

Co-activators (PGC1 $\alpha, \mathrm{PGC1} \beta$ ) and co-repressors (CtBP1, CtBP2, NRIP1) of PPAR $\gamma$ were also expressed, demonstrating the ability of these cells to modulate PPAR signaling pathways. hMADS cells also expressed other classical components of brown adipocyte signaling such as DiO2 (thyroid hormone pathway), COX1, COX2, PGTiS, and PGTiR (arachidonic acid pathway) and interestingly, an increase in the bile acid receptor, GPBAR1.

Altogether, these observations clearly confirmed the "brite" phenotype of hMADS adipocytes as they display both an origin and a molecular signature distinct from those of genuine brown adipocytes.

\section{EFFECTS OF HEDGEHOG AND BMP SIGNALING EFFECTORS ON BRITE hMADS CELL FORMATION}

As conversion of hMADS cells into brite adipocytes is dependent upon PPAR $\gamma$ activation, we became interested in deciphering pathways that are independent of rosiglitazone treatment. It has been shown in mouse models that Hedgehog and BMP pathways modulated the formation of brown adipocytes (Lee et al., 2008; Pospisilik et al., 2010; Schulz et al., 2011). We analyzed UCP1 expression after activation of the Hedgehog pathway, using two known activators of 


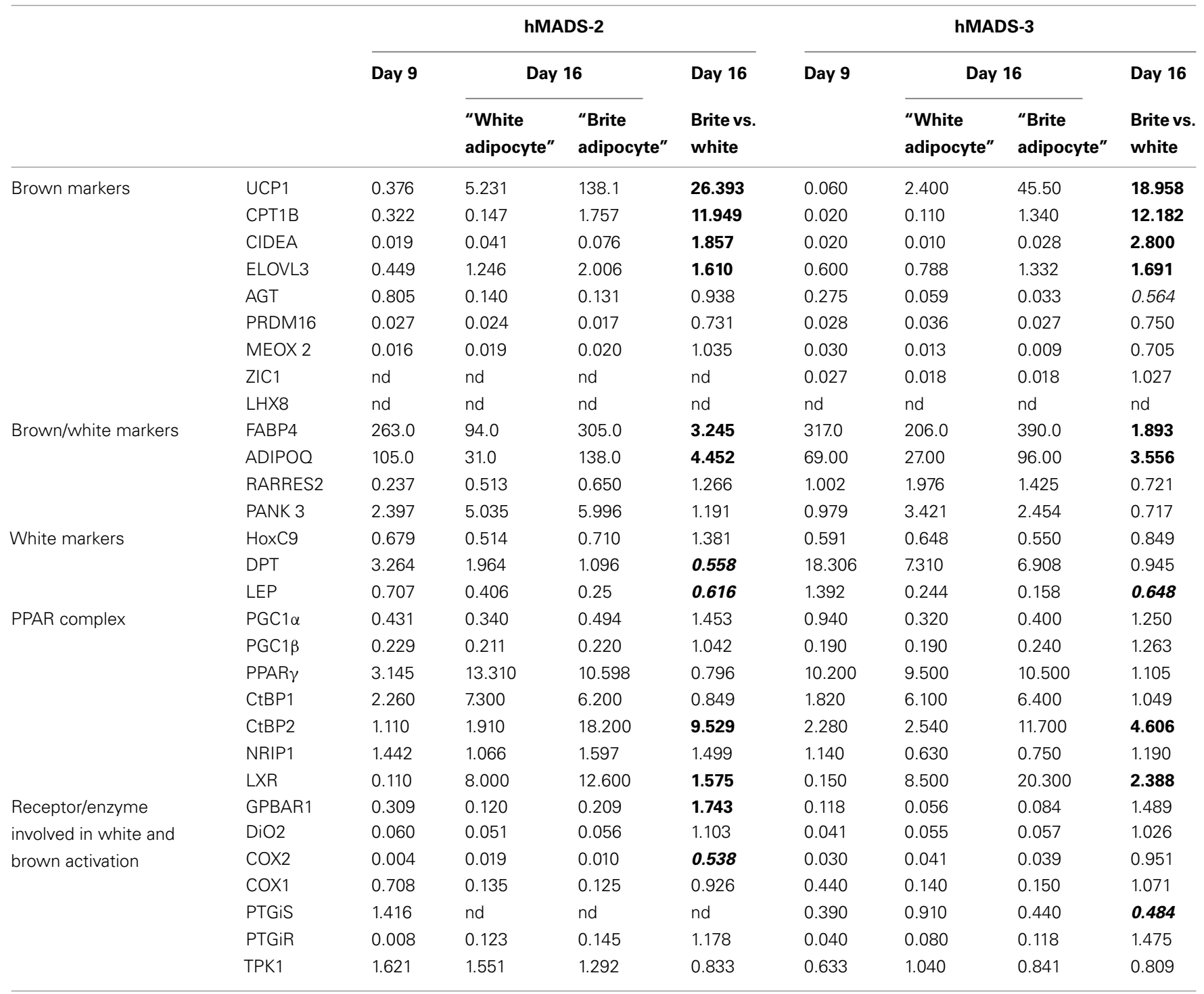

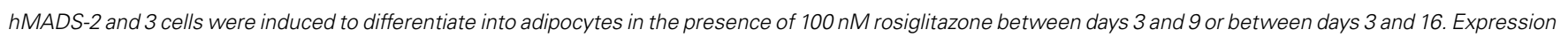
of various markers described previously was analyzed by quantitative RT-PCR. The expression values ( $\triangle C t$ referred to TBP) mRNA in hMADS cells at the indicated. Genes indicated in italics and bold are highly expressed in white adipocyte and in brown adipocyte conditions, respectively.

smoothen, i.e., purmorphamine and smoothened agonist (SAG). hMADS cells that were differentiated in the presence of rosiglitazone either between day 3 and 9 or between day 3 and 16 were exposed to the two compounds and analyzed at day 16. As expected, the expression of Gli1 mRNA (Figure 4A), a marker of the activation of Hedgehog signaling (Hooper and Scott, 2005), was observed. However, neither purmorphamine nor SAG were able to substitute to rosiglitazone and to modulate the expression of UCP1 or FABP4.

In a similar way we tested whether BMPs could induce the "browning" of hMADS cells. hMADS cells, in the presence or absence of rosiglitazone between days 3 and 9, were treated with or without $10 \mathrm{nM}$ BMP2 or BMP7. As shown in Figure 4B, neither BMP2 nor BMP7 affected adipogenesis of hMADS cells, as illustrated by the expression of FABP4 and only induced weakly the formation of brite adipocytes as illustrated by the expression of UCP1 and CIDEA.

\section{DISCUSSION}

Recent advances in the developmental analysis of white and brown progenitors demonstrated a distinct origin (Timmons et al., 2007; Crisan et al., 2008). Furthermore, brown-like adipocytes are found in white fat depots upon physiological or pharmacological stimulation and there is evidence that white adipocytes can be converted to brown-like fat cells (Tiraby et al., 2003; Cinti, 2009a,b). A recent study described mouse "brite" adipocytes, obtained upon chronic PPAR $\gamma$ activation of primary cultures of white adipocyte precursors (Petrovic et al., 2010), which may 
A
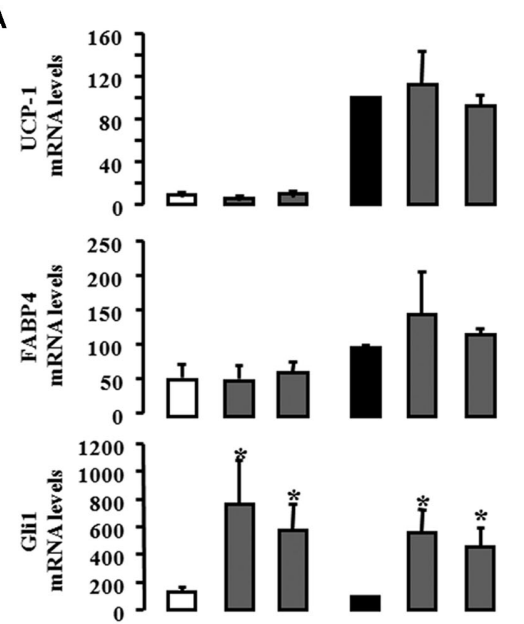

purmo $1 \mu \mathrm{M}$ SAG $1 \mu \mathrm{M}$

]

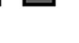

B
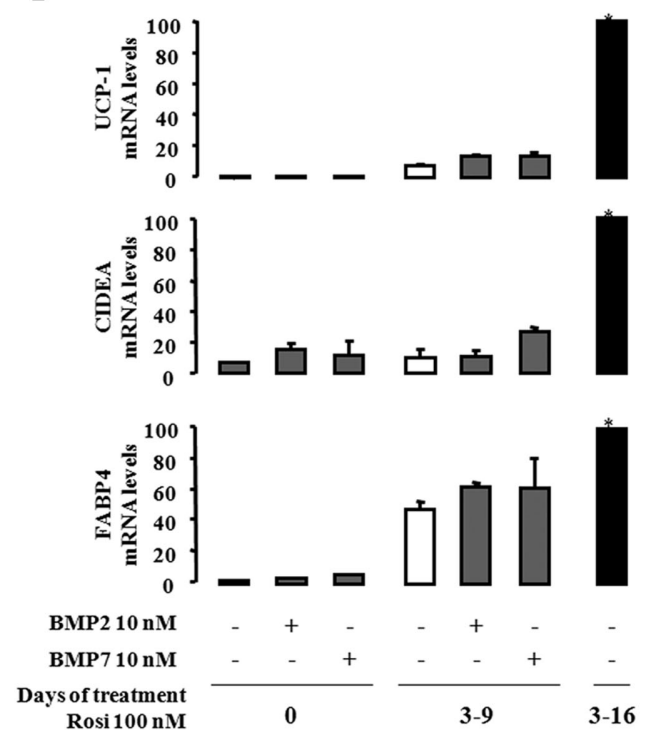

FIGURE 4 | Effect of Hedgehog and BMP signaling in the conversion of white to brown adipocytes. hMADS-2 cells were induced to differentiate into adipocytes in presence of $100 \mathrm{nM}$ rosiglitazone at the indicate times. (A), Purmorphamine (purmo) or smoothened agonist (SAG) as effectors of the Hedgehog signaling pathway were added between days 14 and 18 . Their effects were analyzed by measuring UCP1, FABP4, and Gli1 mRNA expression. (B) hMADS-2 cells were treated or not with $10 \mathrm{nM}$ BMP2 or BMP7 between days 0 and 16, and analyzed at day 16 . Results are the mean \pm SEM of three independent experiments and are expressed by taking as $100 \%$ the value obtained for $100 \mathrm{nM}$ rosiglitazone treatment between days 14 and $18 .{ }^{*} p<0.05$ vs. cells treated only by rosiglitazone $100 \mathrm{nM}$. explain the UCP1 expression found in islets surrounded by white adipocytes (Cousin et al., 1992; Xue et al., 2009). In adult humans, the increase of white to "brite" adipocyte conversion and activity, taking advantage of the important mass of WAT, could represent a novel strategy to combat overweight/obesity as a result of energy imbalance. Unfortunately, detailed investigations are hampered ex vivo by the unavailability of human brite and genuine brown adipocytes.

The observations reported herein emphasize that hMADS cells can differentiate into brite adipocytes. Their differentiation is associated with an increase of mitochondrial markers (Figure 3). During proliferation and differentiation, hMADS cells do not express Myf5 indicating that a common signature with myoblasts can be ruled out. Analyzing the expression pattern of genes known to be associated with brown or brite murine adipocytes, clearly demonstrate the brite signature of converted hMADS cells. Indeed, these cells express brown adipocytes markers (PPAR $\alpha$, CPT1B, ELOVL3, CIDEA, PGC1a, and UCP1) and do not (ZIC1, LHX8) or barely express other specific markers (PRDM16, MEOX2) of genuine mouse brown adipocytes. Moreover, hMADS cells express well-known components of brown adipocyte signaling, demonstrating that these cells are able to respond to various signals such as $\alpha$ - and $\beta$-adrenergic agonists, bile acids, fatty acids and prostaglandins, thyroid hormone, and the natriuretic peptide (Table 2; Rodriguez et al., 2004; Elabd et al., 2009). Therefore, hMADS cells can be used as a human cell model to analyze various brown modulating signals that have been described in rodents. Our results clearly emphasize Hedgehog and
BMPs signaling as striking examples of the discrepancies existing between human and rodents (Fontaine et al., 2008; Svensson et al., 2011). Actually, it has been previously shown that activation of Hedgehog signaling in vivo and in vitro impairs white but not brown adipocyte differentiation (Pospisilik et al., 2010). Herein we showed that treatment of hMADS cells with Hedgehog activators did not affect white or brite adipocyte formation (Figure 4A). Furthermore, our data show that BMP7 induces weakly brite adipocyte formation of hMADS cells, in contrast to earlier observations in mouse cell models (Tseng et al., 2008; Schulz et al., 2011) despite the fact that hMADS cells do express functional BMP receptors (Zaragosi et al., 2010; and data not shown).

Taken together, our data demonstrated that differentiated brown-like hMADS adipocytes are representative of the so-called brite adipocytes recently described in mice (Petrovic et al., 2010) though significant differences are observed with regard to signaling cues favoring brown adipogenesis. In conclusion, our human cell model could contribute to shed some light on the mechanisms involved in the browning process of white adipocytes and could help in identifying drugs involved in this phenomenon.

\section{ACKNOWLEDGMENTS}

This work was supported by the Centre National de la Recherche Scientifique and by grants from "ANR miRBAT" and Nutricia Research Foundation, and by GEN-AU, the Austrian Genome Research (GEN-AU) funding program (grant "non-coding RNAs"; no. 820982). 


\section{REFERENCES}

Ailhaud, G., Grimaldi, P., and Negrel, R. (1992). Cellular and molecular aspects of adipose tissue development. Annu. Rev. Nutr. 12, 207-233.

Barbatelli, G., Murano, I., Madsen, L., Hao, Q., Jimenez, M., Kristiansen, K., Giacobino, J. P., De Matteis, R., and Cinti, S. (2010). The emergence of cold-induced brown adipocytes in mouse white fat depots is determined predominantly by white to brown adipocyte transdifferentiation. Am. J. Physiol. Endocrinol. Metab. 298, E1244-E1253.

Bezy, O., Elabd, C., Cochet, O., Petersen, R. K., Kristiansen, K., Dani, C., Ailhaud, G., and Amri, E. Z. (2005). Delta-interacting protein $\mathrm{A}$, a new inhibitory partner of CCAAT/enhancer-binding protein beta, implicated in adipocyte differentiation. J. Biol. Chem. 280, 11432-11438.

Cinti, S. (2009a). Reversible physiological transdifferentiation in the adipose organ. Proc. Nutr. Soc. 68, 340-349.

Cinti, S. (2009b). Transdifferentiation properties of adipocytes in the adipose organ. Am. J. Physiol. Endocrinol. Metab. 297, E977-E986.

Cousin, B., Cinti, S., Morroni, M., Raimbault, S., Ricquier, D., Penicaud, L., and Casteilla, L. (1992). Occurrence of brown adipocytes in rat white adipose tissue: molecular and morphological characterization. J. Cell. Sci. 103(Pt 4), 931-942.

Crisan, M., Casteilla, L., Lehr, L., Carmona, M., Paoloni-Giacobino, A., Yap, S., Sun, B., Leger, B., Logar, A., Penicaud, L., Schrauwen, P., Cameron-Smith, D., Russell, A. P., Peault, B., and Giacobino, J. P. (2008). A reservoir of brown adipocyte progenitors in human skeletal muscle. Stem Cells 26, 2425-2433.

Cypess, A. M., Lehman, S., Williams, G., Tal, I., Rodman, D., Goldfine, A. B., Kuo, F. C., Palmer, E. L., Tseng, Y. H., Doria, A., Kolodny, G. M., and Kahn, C. R. (2009). Identification and importance of brown adipose tissue in adult humans. N. Engl. J. Med. 360, 1509-1517.

Elabd, C., Chiellini, C., Carmona, M., Galitzky, J., Cochet, O., Petersen, R., Penicaud, L., Kristiansen, K., Bouloumie, A., Casteilla, L., Dani, C., Ailhaud, G., and Amri, E. Z. (2009). Human multipotent adiposederived stem cells differentiate into functional brown adipocytes. Stem Cells 27, 2753-2760.

Elabd, C., Chiellini, C., Massoudi, A., Cochet, O., Zaragosi, L. E., Trojani, C., Michiels, J. F., Weiss, P., Carle,
G., Rochet, N., Dechesne, C. A., Ailhaud, G., Dani, C., and Amri, E. Z. (2007). Human adipose tissuederived multipotent stem cells differentiate in vitro and in vivo into osteocyte-like cells. Biochem. Biophys. Res. Commun. 361, 342-348.

Feldmann, H. M., Golozoubova, V., Cannon, B., and Nedergaard, J. (2009). UCP1 ablation induces obesity and abolishes diet-induced thermogenesis in mice exempt from thermal stress by living at thermoneutrality. Cell Metab. 9, 203-209.

Fontaine, C., Cousin, W., Plaisant, M., Dani, C., and Peraldi, P. (2008). Hedgehog signaling alters adipocyte maturation of human mesenchymal stem cells. Stem Cells 26, 1037-1046.

Fruhbeck, G., Becerril, S., Sainz, N., Garrastachu, P., and Garcia-Velloso, M. J. (2009). BAT: a new target for human obesity? Trends Pharmacol. Sci. 30, 387-396.

Golozoubova, V., Cannon, B., and Nedergaard, J. (2006). UCP1 is essential for adaptive adrenergic nonshivering thermogenesis. Am. J. Physiol. Endocrinol. Metab. 291, E350-E357.

Guerra, C., Koza, R. A., Yamashita, H., Walsh, K., and Kozak, L. P. (1998). Emergence of brown adipocytes in white fat in mice is under genetic control. Effects on body weight and adiposity. J. Clin. Invest. 102, 412-420.

Hooper, J. E., and Scott, M. P. (2005). Communicating with Hedgehogs. Nat. Rev. Mol. Cell Biol. 6, 306-317.

Hossain, P., Kawar, B., and El Nahas, M. (2007). Obesity and diabetes in the developing world - a growing challenge. N. Engl. J. Med. 356, 213-215.

Lee, S. Y., Nakagawa, T., and Reddi, A. H. (2008). Induction of chondrogenesis and expression of superficial zone protein (SZP)/lubricin by mesenchymal progenitors in the infrapatellar fat pad of the knee joint treated with TGF-betal and BMP7. Biochem. Biophys. Res. Commun. 376, 148-153.

Nedergaard, J., Bengtsson, T., and Cannon, B. (2007). Unexpected evidence for active brown adipose tissue in adult humans. Am. J. Physiol. Endocrinol. Metab. 293, E444-E452.

Negrel, R., Grimaldi, P., and Ailhaud, G. (1978). Establishment of preadipocyte clonal line from epididymal fat pad of ob/ob mouse that responds to insulin and to lipolytic hormones. Proc. Natl. Acad. Sci. U.S.A. 75, 6054-6058.

Petrovic, N., Walden, T. B., Shabalina, I. G., Timmons, J. A., Cannon, B., and Nedergaard, J. (2010). Chronic peroxisome proliferator-activated receptor gamma (PPARgamma) activation of epididymally derived white adipocyte cultures reveals a population of thermogenically competent, UCP1-containing adipocytes molecularly distinct from classic brown adipocytes. J. Biol. Chem. 285, 7153-7164.

Pisani, D. F., Dechesne, C. A., Sacconi, S., Delplace, S., Belmonte, N., Cochet, O., Clement, N., Wdziekonski, B., Villageois, A. P., Butori, C., Bagnis, C., Di Santo, J. P., Kurzenne, J. Y., Desnuelle, C., and Dani, C. (2010). Isolation of a highly myogenic CD34-negative subset of human skeletal muscle cells free of adipogenic potential. Stem Cells 28, 753-764.

Pospisilik, J. A., Schramek, D., Schnidar, H., Cronin, S. J., Nehme, N. T., Zhang, X., Knauf, C., Cani, P. D., Aumayr, K., Todoric, J., Bayer, M., Haschemi, A., Puviindran, V., Tar, K., Orthofer, M., Neely, G. G., Dietzl, G., Manoukian, A., Funovics, M., Prager, G., Wagner, O., Ferrandon, D., Aberger, F., Hui, C. C., Esterbauer, H., and Penninger, J. M. (2010). Drosophila genome-wide obesity screen reveals hedgehog as a determinant of brown versus white adipose cell fate. Cell 140, 148-160.

Rodriguez, A. M., Elabd, C., Amri, E. Z., Ailhaud, G., and Dani, C. (2005a). The human adipose tissue is a source of multipotent stem cells. Biochimie $87,125-128$.

Rodriguez, A. M., Pisani, D., Dechesne, C. A., Turc-Carel, C., Kurzenne, J. Y., Wdziekonski, B., Villageois, A., Bagnis, C., Breittmayer, J. P., Groux, H., Ailhaud, G., and Dani, C. (2005b). Transplantation of a multipotent cell population from human adipose tissue induces dystrophin expression in the immunocompetent mdx mouse. J. Exp. Med. 201, 1397-1405.

Rodriguez, A. M., Elabd, C., Delteil, F., Astier, J., Vernochet, C., Saint-Marc, P., Guesnet, J., Guezennec, A., Amri, E. Z., Dani, C., and Ailhaud, G. (2004). Adipocyte differentiation of multipotent cells established from human adipose tissue. Biochem. Biophys. Res. Commun. 315, 255-263.

Rosen, E. D., and Spiegelman, B. M. (2006). Adipocytes as regulators of energy balance and glucose homeostasis. Nature 444, 847-853.

Saito, M., Okamatsu-Ogura, Y., Matsushita, M., Watanabe, K., Yoneshiro, T., Nio-Kobayashi, J., Iwanaga, T., Miyagawa, M., Kameya, T., Nakada, K., Kawai, Y., and Tsujisaki, M. (2009). High incidence of metabolically active brown adipose tissue in healthy adult humans: effects of cold exposure and adiposity. Diabetes 58, 1526-1531.

Schulz, T. J., Huang, T. L., Tran, T. T., Zhang, H., Townsend, K. L., Shadrach, J. L., Cerletti, M., Mcdougall, L. E., Giorgadze, N., Tchkonia, T., Schrier, D., Falb, D., Kirkland, J. L., Wagers, A. J., and Tseng, Y. H. (2011). Identification of inducible brown adipocyte progenitors residing in skeletal muscle and white fat. Proc. Natl. Acad. Sci. U.S.A. 108, 143-148.

Seale, P., Bjork, B., Yang, W., Kajimura, S., Chin, S., Kuang, S., Scime, A., Devarakonda, S., Conroe, H. M., Erdjument-Bromage, H., Tempst, P., Rudnicki, M. A., Beier, D. R., and Spiegelman, B. M. (2008). PRDM16 controls a brown fat/skeletal muscle switch. Nature 454, 961-967.

Svensson, P. A., Jernas, M., Sjoholm, K., Hoffmann, J. M., Nilsson, B. E., Hansson, M., and Carlsson, L. M. (2011). Gene expression in human brown adipose tissue. Int. J. Mol. Med. 27, 227-232.

Timmons, J. A., Wennmalm, K., Larsson, O., Walden, T. B., Lassmann, T., Petrovic, N., Hamilton, D. L., Gimeno, R. E., Wahlestedt, C., Baar, K., Nedergaard, J., and Cannon, B. (2007). Myogenic gene expression signature establishes that brown and white adipocytes originate from distinct cell lineages. Proc. Natl. Acad. Sci. U.S.A. 104, 4401-4406.

Tiraby, C., Tavernier, G., Lefort, C., Larrouy, D., Bouillaud, F., Ricquier, D., and Langin, D. (2003). Acquirement of brown fat cell features by human white adipocytes. J. Biol. Chem. 278, 33370-33376.

Tseng, Y. H., Kokkotou, E., Schulz, T. J., Huang, T. L., Winnay, J. N., Taniguchi, C. M., Tran, T. T., Suzuki, R., Espinoza, D. O., Yamamoto, Y., Ahrens, M. J., Dudley, A. T., Norris, A. W., Kulkarni, R. N., and Kahn, C. R. (2008). New role of bone morphogenetic protein 7 in brown adipogenesis and energy expenditure. Nature 454, 1000-1004.

Van Marken Lichtenbelt, W. D., Vanhommerig, J. W., Smulders, N. M., Drossaerts, J. M., Kemerink, G. J., Bouvy, N. D., Schrauwen, P., and Teule, G. J. (2009). Coldactivated brown adipose tissue in healthy men. N. Engl. J. Med. 360, 1500-1508.

Vernochet, C., Peres, S. B., Davis, K. E., Mcdonald, M. E., Qiang, L., Wang, H., Scherer, P. E., and Farmer, S. R. (2009). C/EBPalpha and the corepressors CtBP1 and 
Pisani et al.

Conversion of hMADS cells into brite adipocytes

CtBP2 regulate repression of select visceral white adipose genes during induction of the brown phenotype in white adipocytes by peroxisome proliferator-activated receptor gamma agonists. Mol. Cell. Biol. 29, 4714-4728.

Virtanen, K. A., Lidell, M. E., Orava, J., Heglind, M., Westergren, R., Niemi, T., Taittonen, M., Laine, J., Savisto, N. J., Enerback, S., and Nuutila, P. (2009). Functional brown adipose tissue in healthy adults. N. Engl. J. Med. 360, 1518-1525.

Whittle, A. J., Lopez, M., and Vidal-Puig, A. (2011). Using brown adipose tissue to treat obesity - the central issue. Trends Mol. Med. 17, 405-411.

Xue, B., Rim, J. S., Hogan, J. C., Coulter, A. A., Koza, R. A., and Kozak, L. P. (2007). Genetic variability affects the development of brown adipocytes in white fat but not in interscapular brown fat. J. Lipid Res. 48, 41-51.

Xue, Y., Petrovic, N., Cao, R., Larsson, O., Lim, S., Chen, S., Feldmann, H. M., Liang, Z., Zhu, Z., Nedergaard, J., Cannon, B., and Cao, Y. (2009). Hypoxia-independent angiogenesis in adipose tissues during cold acclimation. Cell Metab. 9, 99-109.

Zaragosi, L. E., Ailhaud, G., and Dani, C. (2006). Autocrine fibroblast growth factor 2 signaling is critical for self-renewal of human multipotent adipose-derived stem cells. Stem Cells 24, 2412-2419.

Zaragosi, L. E., Wdziekonski, B., Villageois, P., Keophiphath, M., Maumus, M., Tchkonia, T., Bourlier, V., Mohsen-Kanson, T., Ladoux, A., Elabd, C., Scheideler, M., Trajanoski, Z., Takashima, Y., Amri, E. Z., Lacasa, D., Sengenes, C., Ailhaud, G.,
Clement, K., Bouloumie, A., Kirkland, J. L., and Dani, C. (2010). Activin a plays a critical role in proliferation and differentiation of human adipose progenitors. Diabetes 59, 2513-2521.

Zingaretti, M. C., Crosta, F., Vitali, A., Guerrieri, M., Frontini, A., Cannon, B., Nedergaard, J., and Cinti, S. (2009). The presence of UCP1 demonstrates that metabolically active adipose tissue in the neck of adult humans truly represents brown adipose tissue. FASEB J. 23, 3113-3120.

Conflict of Interest Statement: The authors declare that the research was conducted in the absence of any commercial or financial relationships that could be construed as a potential conflict of interest.
Received: 29 July 2011; accepted: 12 November 2011; published online: 29 November 2011.

Citation: Pisani DF, Djedaini $M$, Beranger GE, Elabd C, Scheideler $M$, Ailhaud G and Amri E-Z (2011) Differentiation of human adipose-derived stem cells into "brite" (brown-in-white) adipocytes. Front. Endocrin. 2:87. doi: 10.3389/fendo.2011.00087

This article was submitted to Frontiers in Cellular Endocrinology, a specialty of Frontiers in Endocrinology.

Copyright (C) 2011 Pisani, Djedaini, Beranger, Elabd, Scheideler, Ailhaud and Amri. This is an open-access article distributed under the terms of the Creative Commons Attribution Non Commercial License, which permits use, distribution, and reproduction in other forums, provided the original authors and source are credited.

www.frontiersin.org

November 2011 | Volume 2 | Article 87 | 9 